\title{
GARANTÍAS EXPLÍCITAS EN SALUD DEL PROGRAMA CÁNCER CERVICOUTERINO: GRADO DE ACUERDO DE LOS PROFESIONALES DEL SERVICIO DE SALUD METROPOLITANO SUR ORIENTE *
}

\author{
María Teresa Urrutia S. ${ }^{1 a}$, Natalia Villegas R. ${ }^{a}$, Lauren Poupin B. ${ }^{a}$ \\ 1 Escuela de Enfermería, Facultad de Medicina, Pontificia Universidad Católica de Chile \\ a Enfermera-Matrona \\ * Proyecto FONIS, SA05I20047, CONICYT, MINSAL, Chile.
}

\section{RESUMEN}

Antecedentes: El cáncer cérvicouterino (CC) es una de las principales causas de muerte por cáncer en la mujer en países subdesarrollados. En Chile el programa $\mathrm{CaCu}$ ha sido una de las prioridades en salud, siendo incorporado al sistema AUGE. Objetivo: Conocer el grado de acuerdo de los profesionales del programa CC, con las guías clínicas, las garantías explicitas en salud y el sistema de registro del programa. Método: Estudio descriptivo transversal, con una muestra de 59 profesionales del Servicio de Salud Metropolitano Sur-Oriente de la ciudad de Santiago provenientes de los tres niveles de atención del programa AUGE-CC. Resultados: Promedio de edad fue de 39,2 \pm 10,3 años. El tiempo promedio en el cargo es de $7,7 \pm 7,4$ años. Más del $90 \%$ está de acuerdo con la garantía de acceso, sobre el $66 \%$ con la garantía de oportunidad y $59,3 \%$ con la garantía financiera. El $94,4 \%$ refiere conocer las guías clínicas y más del $65 \%$ estuvo de acuerdo con ellas. El $32 \%$ de los profesionales está de acuerdo con el sistema de registros vigente. Conclusiones: Es alto el grado de acuerdo de los profesionales con la garantía de acceso, menor la de oportunidad y más baja con la garantía financiera. Se considera importante considerar la opinión de los profesionales involucrados en el sistema para mejorar el funcionamiento del programa.

\section{PALABRAS CLAVE: Cáncer cervicouterino, garantías explícitas en salud (GES), reforma de salud}

\section{SUMMARY}

Background: Cervical cancer (CC) is a leading cause of cancer death among women from developing countries. In Chile the Cervical Cancer Program has been one of the priorities in health, being incorporated to the AUGE system. Objective: To identify the level of agreement among professionals from the CC program regarding the CC clinical guide's norms, explicit guaranties in health and the program's registration system. Methods: Cross-sectional descriptive study with a sample of 59 professionals in the Metropolitan South-East Health Service in Santiago, from the three levels of care. Results: The average age was $39.2 \pm 10.3$ years. The average time in their job positions was $7.7 \pm 7.4$ years. Among professionals, more than $90 \%$ agree with the guarantee for access, $66 \%$ with the guarantee for opportunity and $59.3 \%$ with the financial guarantee. Additionally, $94.4 \%$ reported they were familiar with the clinical guide and $65 \%$ reported they agree with it. Thirty two percent of professionals agree with the existing registration system. Conclusions: The perception 
of agreement among professionals is high regarding the guarantee for access, lower regarding the guarantee for opportunity, and the lowest regarding the financial guarantee. It is important to consider the health care's perception for improving the operation of the program.

\section{KEY WORDS: Cervical cancer, explicit health guarantees (GES), health reform}

\section{INTRODUCCIÓN}

El cáncer cervicouterino (CC) es una de las principales causas de muerte por cáncer en Latinoamérica. Globalmente se estima 470.606 casos nuevos y 233.372 defunciones por $\mathrm{CC}$ en el mundo, correspondiendo el $80 \%$ a países menos desarrollados (1,2). En Chile, el CC es la primera causa de muerte en las mujeres de 20 a 44 años y la sexta en el grupo de 45 a 64 años (3).

En nuestro país el CC ha sido una prioridad en salud, lo que se vio reflejado al ser incorporado al proceso de puesta en marcha del sistema AUGE (Acceso Universal Garantías Explícitas) en el año 2003. Las Garantías Explicitas en Salud (GES) corresponden a "un derecho que otorga la ley a todos los afiliados a FONASA e ISAPRES y a sus respectivas cargas, para ser atendidos en condiciones especiales que garantizan su acceso, calidad, oportunidad y protección financiera en caso de sufrir cualquiera de los problemas de salud definidos por el Ministerio de Salud" (4).

El objetivo de este estudio es conocer el grado de acuerdo que tienen los profesionales del programa CC-AUGE, con las condiciones establecidas en las guías clínicas para CC, las GES del programa y el sistema de registro.

\section{MATERIAL Y MÉTODO}

Estudio descriptivo de corte transversal en un grupo de profesionales del Servicio de Salud Metropolitano Sur Oriente (SSMSO). La muestra está constituida por profesionales del programa CCAUGE de los tres niveles de atención: en el nivel primario el profesional matrón(a) responsable del programa CC de cada consultorio, en el nivel secundario al médico y matrón(a) del policlínico de la especialidad de cada centro hospitalario (Complejo Asistencial Dr. Sótero del Río y Hospital Padre Alberto Hurtado), y en el nivel terciario al médico y matrón(a) de los servicios de ginecología oncológica de los dos centros hospitalarios, y los profesionales del Instituto Nacional del Cáncer, donde se realiza el tratamiento de radioterapia a algunas de las usuarias con lesión invasora. La muestra corresponde a un total de 59 profesionales, lo que equivale al $98 \%$ del universo ya que solo 1 profesional no contestó el cuestionario. Los criterios de inclusión fueron: ser profesional que trabaja en los centros hospitalarios señalados o consultorios respectivos, estar en atención directa con la usuaria del programa CC-AUGE y estar en ejercicio del cargo por lo menos 6 meses previo a la aplicación del cuestionario.

La recolección de los datos fue realizada durante el periodo comprendido entre mayo y octubre de 2006. Las variables a medir fueron: características de los profesionales (edad, profesión, especialidad, lugar de trabajo y tiempo en el cargo), grado de acuerdo con las guías clínicas CC-AUGE (escala de Likert 5 alternativas), acuerdo con las GES establecidas en las guías clínicas (Si-No-No sabe), y grado de acuerdo con el sistema de registro vigente (Si-Parcialmente-No). Se solicitó señalar aspectos positivos y negativos del sistema de registro y proponer sugerencias. Esta investigación contó con la aprobación de los comités de ética: de la Escuela de Enfermería de la Pontificia Universidad Católica de Chile y del SSMSO. Se solicitó firmar voluntariamente el consentimiento informado a cada uno de los profesionales del estudio. Los datos se analizaron con el programa SPSS 16.0 y se utilizaron medidas de tendencia central, de dispersión y análisis de frecuencia.

\section{RESULTADOS}

Características de los profesionales. El promedio de edad de los profesionales fue de 39,2 $\pm 10,3$ años, con un rango de 25 a 70 años. Del total de la muestra 45 son matronas(es), 13 médicos y 1 enfermera. El $69,5 \%$ trabaja en el nivel primario, el $6,8 \%$ en el nivel secundario, el $3,4 \%$ en el nivel secundario y terciario, y el $20,4 \%$ en el nivel terciario. El tiempo promedio en el cargo es $7,7 \pm 7,4$ años con un rango de 6 meses a 34 años. El 22\% de la muestra refiere tener una especialización en oncología, con un período de formación entre 1,5 a 4 años; todos ellos corresponde a profesionales médicos. 
Guía clínica. El 94,4\% de los profesionales refiere conocer la guía clínica CC-AUGE, el 3,4\% dice conocerla parcialmente y sólo un 1,7\% ( $n=1)$ menciona que no la conoce. Más del $65 \%$ de los profesionales estuvo de acuerdo, con alguno de los 11 ítems referentes a las normas clínicas para CC. En la Tabla I, se presentan los resultados de cada uno de los aspectos evaluados de las guías clínicas.

Grado de acuerdo con la garantía de acceso: El grado de acuerdo con la garantía de acceso es superior al $90 \%$. En la Tabla II se observa la distribución de las respuestas de acuerdo a las diferentes etapas y plazos que incluye el algoritmo de atención.

Tabla I

GRADO DE ACUERDO DE LOS PROFESIONALES CON LAS NORMAS ESTABLECIDAS EN LAS GUÍAS CLÍNICAS PARA CC-AUGE

\begin{tabular}{|c|c|c|c|c|c|c|}
\hline \multirow{2}{*}{$\begin{array}{l}\text { Grado de acuerdo } \\
\text { Escala de Likert }\end{array}$} & \multicolumn{2}{|c|}{$\begin{array}{l}\text { Completamente en } \\
\text { desacuerdo: } \mathrm{n}(\%)\end{array}$} & \multirow{2}{*}{$\frac{n(\%)}{3}$} & \multicolumn{2}{|c|}{$\begin{array}{l}\text { Completamente en } \\
\text { acuerdo: } n(\%)\end{array}$} & \multirow[t]{2}{*}{$\mathrm{n}$} \\
\hline & 1 & 2 & & 4 & 5 & \\
\hline 1. Objetivos & - & - & - & $14(23,7)$ & $42(71,2)$ & 56 \\
\hline $\begin{array}{l}\text { 2. Algoritmo establecido para la } \\
\text { red de atención desde la pesquisa } \\
\text { hasta el tratamiento }\end{array}$ & - & - & $4(6,8)$ & 13(22) & $39(66,1)$ & 56 \\
\hline $\begin{array}{l}\text { 3.Intervenciones recomendadas } \\
\text { para la confirmación diagnóstica }\end{array}$ & - & - & $1(1,7)$ & $9(15,3)$ & $45(76,3)$ & 55 \\
\hline $\begin{array}{l}\text { 4. Algoritmo para la confirmación } \\
\text { diagnóstica }\end{array}$ & - & - & - & $10(16,9)$ & $45(76,3)$ & 55 \\
\hline $\begin{array}{l}\text { 5. Intervenciones recomendadas } \\
\text { para la etapificación del cáncer }\end{array}$ & - & - & - & $10(16,9)$ & $43(72,9)$ & 53 \\
\hline $\begin{array}{l}\text { 6. Intervenciones recomendadas } \\
\text { para el tratamiento en general }\end{array}$ & - & - & $1(1,7)$ & $10(16,9)$ & $44(74,6)$ & 55 \\
\hline $\begin{array}{l}\text { 7. Intervenciones recomendadas } \\
\text { para el tratamiento de lesiones } \\
\text { pre invasoras }\end{array}$ & - & - & $2(3,4)$ & $11(18,6)$ & $41(69,5)$ & 54 \\
\hline $\begin{array}{l}\text { 8. Tratamiento de lesiones } \\
\text { invasoras }\end{array}$ & - & - & - & $8(13,6)$ & $45(76,3)$ & 53 \\
\hline $\begin{array}{l}\text { 9. Intervenciones recomendadas } \\
\text { para el seguimiento }\end{array}$ & - & - & $1(1,7)$ & 13(22) & $41(69,5)$ & 55 \\
\hline $\begin{array}{l}\text { 10. Intervenciones recomendadas } \\
\text { para la recidiva }\end{array}$ & $1(1,7 \%)$ & $1(1,7 \%)$ & $3(5,1)$ & $8(13,6)$ & $42(71,2)$ & 55 \\
\hline 11. Criterios de egreso & - & $1(1,7 \%)$ & $1(1,7)$ & $14(23,7)$ & $39(66,1)$ & 55 \\
\hline
\end{tabular}


Tabla II

GRADO DE ACUERDO DE LOS PROFESIONALES CON LA GARANTÍA DE ACCESO DE LAS MUJERES BENEFICIARIAS DEL PLAN CC-AUGE $(n=59)$

\begin{tabular}{|c|c|c|c|c|}
\hline \multirow[b]{2}{*}{ Etapas de la atención } & \multirow[b]{2}{*}{ Garantía de acceso } & \multicolumn{3}{|c|}{ Grado de Acuerdo (\%) } \\
\hline & & De acuerdo & En desacuerdo & No sabe \\
\hline $\begin{array}{l}\text { Con sospecha clínica o } \\
\text { PAP positivo }\end{array}$ & Diagnóstico & 98,3 & - & 1,7 \\
\hline \multirow{2}{*}{$\begin{array}{l}\text { Con confirmación } \\
\text { diagnóstica }\end{array}$} & Tratamiento & 98,3 & - & 1,7 \\
\hline & Seguimiento & 98,3 & - & 1,7 \\
\hline \multirow[t]{3}{*}{ Con recidiva } & Diagnóstico & 94,9 & - & 5,1 \\
\hline & Tratamiento & 94,9 & 1,7 & 3,4 \\
\hline & Seguimiento & 93,2 & - & 6,8 \\
\hline
\end{tabular}

Grado de acuerdo con la garantía de oportunidad: El grado de acuerdo de los profesionales con la garantía de oportunidad y por ende con los plazos establecidos en la lesión pre invasora estuvo entre un $76 \%$ y $88 \%$, y en el caso de lesión invasora, entre un $66 \%$ y $85 \%$. En la Tabla III se observa la distribución de las respuestas de acuerdo a las diferentes etapas y plazos que incluye el algoritmo de atención. En relación con los profesionales que no estuvieron de acuerdo con la garantía de oportunidad, fueron mencionadas diversas razones (Tabla IV). El mayor desacuerdo se encuentra relacionado a los tiempos establecidos, y la preocupación de los profesionales por el estado emocional de las pacientes en las diferentes etapas establecidas en la GES.

Tabla III

GRADO DE ACUERDO DE LOS PROFESIONALES CON LA GARANTÍA DE OPORTUNIDAD DEL PLAN CC-AUGE $(n=59)$

\begin{tabular}{|c|c|c|c|c|}
\hline \multirow[b]{2}{*}{ Etapas de la atención } & \multirow[b]{2}{*}{ Garantía de oportunidad } & \multicolumn{3}{|c|}{ Grado de Acuerdo (\%) } \\
\hline & & De acuerdo & En desacuerdo & No sabe \\
\hline \multicolumn{5}{|l|}{ Lesión preinvasora: } \\
\hline $\begin{array}{l}\text { Con sospecha clínica o } \\
\text { PAP positivo }\end{array}$ & Atención de especialista* & 76,3 & 23,7 & - \\
\hline Atención especialista & Confirmación diagnóstica* & 78,0 & 22,0 & - \\
\hline Confirmación diagnóstica & Tratamiento* & 78,0 & 22,0 & - \\
\hline Fin de tratamiento & Seguimiento* & 88,1 & 11,9 & - \\
\hline \multicolumn{5}{|l|}{ Lesión invasora: } \\
\hline $\begin{array}{l}\text { Con sospecha clínica o } \\
\text { PAP positivo }\end{array}$ & Atención de especialista* & 72,9 & 25,4 & 1,7 \\
\hline Atención especialista & Confirmación diagnóstica* & 71,2 & 27,1 & 1,7 \\
\hline Confirmación diagnóstica & Etapificación ${ }^{\star \star}$ & 76,3 & 22,0 & 1,7 \\
\hline Etapificación & Tratamiento*** & 66,1 & 32,2 & 1,7 \\
\hline Fin de tratamiento & Seguimiento* & 84,5 & 13,6 & 1,7 \\
\hline
\end{tabular}

* 30 días. ${ }^{* \star} 15$ días. ${ }^{\star \star *} 20$ días 


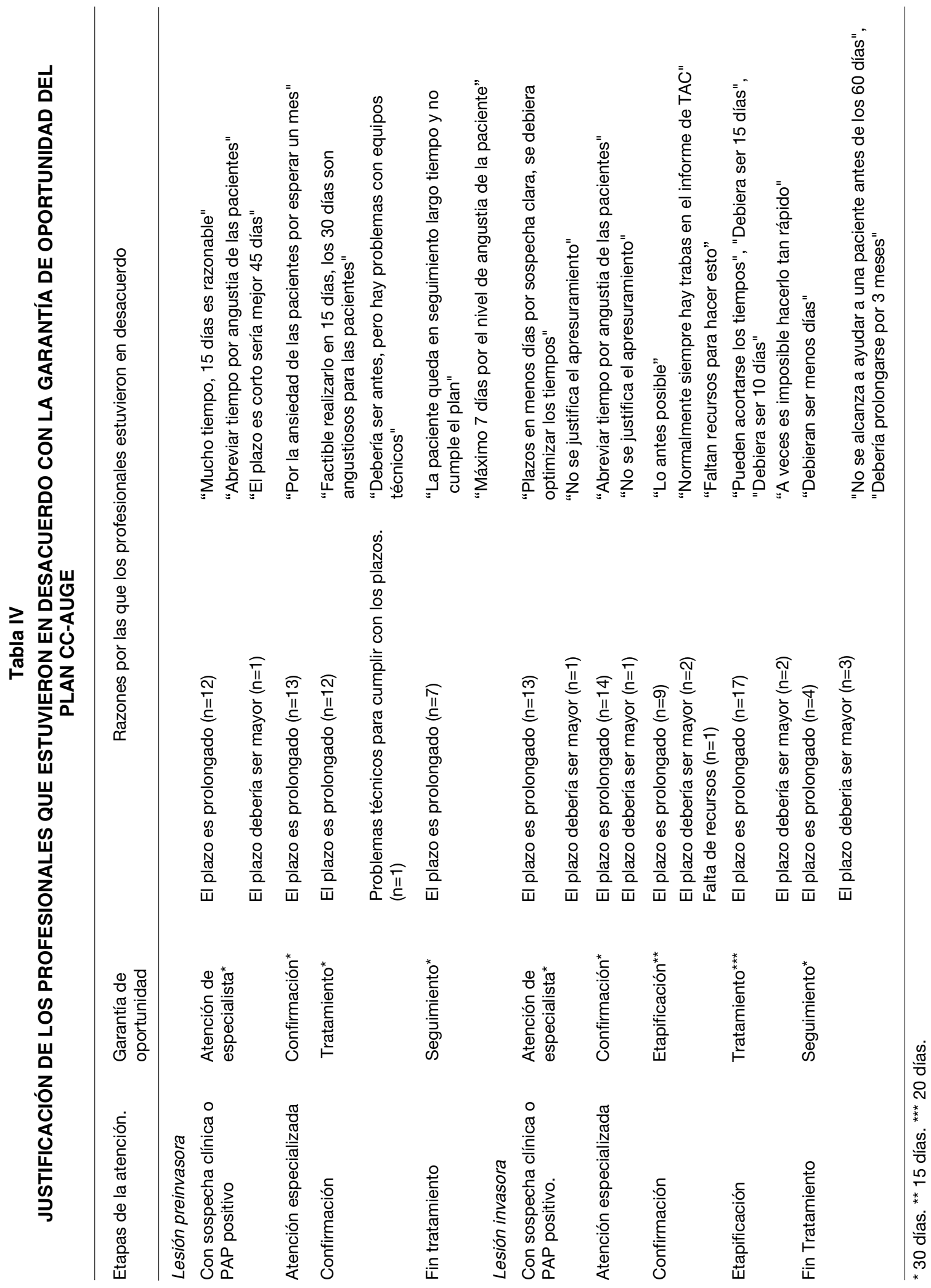


Grado de acuerdo con la garantía financiera: En esta garantía el $59,3 \%$ de los profesionales está de acuerdo, el $18,6 \%$ en desacuerdo y un $22 \%$ de los profesionales no conocen la garantía. Los profesionales que estuvieron en desacuerdo con la garantía financiera mencionaron las siguientes razones: debería ser sin tope; debería hacerse una clasificación social, ya que la clasificación FONASA no corresponde siempre a la realidad; existe una incorrecta valorización del tratamiento; existe dificultad de las mujeres para realizar el copago y cubrir gastos asociados a la enfermedad (por ejemplo la movilización), y para FONASA C y D los copagos son muy altos. Al preguntarles a los profesionales si ellos creían que la cobertura financiera se ajustaba a los costos de la patología sólo un $23,7 \%$ cree que si se ajusta, el $27,1 \%$ manifestó que no creía que se ajustaba y un $49,2 \%$ no sabe. Los profesionales que consideraron que la cobertura financiera no se ajustaba a los costos manifestaron: "algunas prestaciones dentro de la GES no están codificadas (por ejemplo la braquiterapia)", "el costo real de la GES es mucho mayor", "hay errores en la valoración y por ende en lo que FONASA paga al servicio".

Grado de acuerdo con la garantía de calidad: La garantía de calidad de atención no estaba vigente al momento del presente estudio, sin embargo se solicitó a los profesionales, basado en el propósito de la reforma, que sugirieran como medir la garantía de calidad. Los profesionales sugieren medirla a través de indicadores del grado de satisfacción de las usuarias con el sistema (como encuestas de satisfacción usuaria y hojas de reclamos o felicitaciones), acreditación de los profesionales, evaluación continua de las diferentes garantías y su cumplimiento del programa CC-AUGE, conocer la etapa en que se realiza el diagnóstico, evaluar la calidad técnica de los equipos, evaluación del sistema de registro y protocolos. También sugirieron la medición de indicadores epidemiológicos, como por ejemplo porcentaje de sobrevida, recidiva y mortalidad.

Sistema de registro. El $32 \%$ de los profesionales estuvo de acuerdo con el sistema de registro, $37,3 \%$ parcialmente y $22 \%$ no estuvo de acuerdo. El $3,4 \%$ de los profesionales no responde y el $5,1 \%$ dice no conocer el sistema de registros. Dentro de los aspectos positivos se encuentra que es un sistema ordenado y de fácil utilización. Dentro de los aspectos negativos es que es un sistema computacional no vigente, y que no está disponible en red. Las sugerencias para mejorar el sistema de registros incluyen que se actualice el sistema computacional actual; que el sistema sea instalado en línea (idealmente a nivel nacional) para permitir la retroa- limentación entre los niveles de atención, y la navegación de la mujer en el sistema de salud sin perder los antecedentes; que el sistema de registro computacional sea accesible a todos los profesionales de los diferentes niveles de atención, y finalmente que se entregue una adecuada capacitación para el adecuado manejo del programa computacional.

\section{DISCUSIÓN}

Las características socio demográficas de los profesionales encargados de la atención de las usuarias reflejan un rango amplio de edad y años de servicio. El nivel de atención que cuenta con mayor número de profesionales, en este programa, es el nivel primario. Dada las características de esta patología y el enfoque planteado en los objetivos sanitarios (5), es fundamental la prevención primaria y la pesquisa oportuna, ambas actividades realizadas fundamentalmente en el nivel primario de atención, por lo que existe coherencia entre el número de profesionales en dicho nivel. Es importante destacar el bajo porcentaje de entrevistados con un grado de especialización en oncología, siendo solo médicos. Este hecho probablemente guarda relación con la política de capacitación formal exigida en el sistema de salud, además del mayor porcentaje de profesionales que trabaja en el nivel primario de atención, donde el énfasis está en la promoción y prevención y no en el trabajo especializado $(5,6)$.

En cuanto a las GES, el grado de acuerdo con la garantía de acceso, oportunidad y con las normas clínicas de atención establecidas en la guía clínica resultó ser alto; probablemente asociado a que los profesionales concuerdan con los criterios sanitarios incorporados en las diferentes etapas del algoritmo de atención, los cuales están basados en la mejor evidencia disponible. Cabe recordar que para la elaboración de las normas de las guías clínicas se utilizaron recomendaciones basadas en evidencia internacional, con el propósito de otorgar una atención de salud que genere beneficios por sobre los riesgos en las intervenciones realizadas y que además permitan la costo eficacia de éstas (7). Esto podría explicar el porqué estos criterios son compartidos por los profesionales.

La garantía de oportunidad, fue la que menor acuerdo obtuvo por parte de los profesionales, y es quizás la que más polémica puede causar, dado que se exigen plazos para el cumplimiento de determinadas actividades. A pesar de que sobre el $65 \%$ estuvo de acuerdo con la garantía de oportunidad en lesiones preinvasoras e invasoras, esta lectura de resultados debe hacerse con precaución, princi- 
palmente al analizar el reporte de los profesionales que no estuvieron de acuerdo con esta garantía. Las respuestas en desacuerdo con la garantía, reflejan aspectos que son posibles de mejorar en esta GES, como por ejemplo el reducir algunos de los plazos estipulados en el algoritmo de atención, los cuales son definidos como extensos, causando angustia a las pacientes. Cabe señalar que todos los profesionales llevan más de 6 meses en el cargo, por lo que todos ellos han vivido la experiencia de atención de pacientes y el cumplimiento o no de dicha garantía.

Lo anterior plantea un punto importante, ya que si bien existe evidencia para fundamentar el porqué de los criterios de acceso y oportunidad de las GES-CC, muchas veces la puesta en práctica puede ser factible o necesaria de mejorar para alcanzar mejores estándares de atención, en especial en esta GES en donde los mismos profesionales señalaron la necesidad de hacerlo. Esto llama a consideración la creciente necesidad de una fluida comunicación en la red GES, en donde se produzca un intercambio de experiencias, conocimientos y trabajo en conjunto entre los profesionales en todos los niveles que permitan cumplir y mejorar los estándares propuestos e identificar las necesidades actuales del sistema $(5,8,9)$.

En relación, al grado de acuerdo con la garantía financiera, si se ajusta o no a los costos de la patología, un grupo de estos profesionales que esta en desacuerdo percibe que la cobertura es deficiente porque no refleja los costos reales de la patología, lo que hace que exista incertidumbre respecto a la sustentación económica de la patología, y por lo tanto a la credibilidad del programa financieramente hablando. Esta incertidumbre puede ser interpretada en el sentido de que existen limitaciones para lograr una estimación exacta de los costos del financiamiento. Cabe señalar que el sistema para estimar los costos no está totalmente implementado, ni existe un sistema de información uniforme para estimar todos los costos involucrados(10). Este aspecto requiere ser mejorado para verificar la real validez de estos costos, y por lo tanto para asegurar la cobertura según lo plantea la ley GES (11).

Por otra parte el desconocimiento acerca del financiamiento en un grupo de los profesionales apunta a la necesidad de informar y explicar cómo se realiza el cálculo de los costos de las prestaciones y la solidaridad en el sistema. Al respecto cabe señalar que los costos de provisión de las GES se calculan en relación a un beneficiario promedio, considerando ambos aseguradores (FONASA e ISAPRE) y utilizando las prestaciones especifica- das por el MINSAL $(10,11)$. Estos costos deben ser entendidos como el resultado final del problema de salud, las prestaciones garantizadas, el número de casos esperados y la demanda potencial que exista en las prestaciones de ese problema de salud (10). El principio de solidaridad en el sistema, consiste en que una parte de la población debe financiar una proporción o la totalidad de los costos de los servicios de salud que consume otro grupo (12), existiendo una "redistribución entre quienes (sanos y jóvenes) tienen más recursos y/o menos riesgo y aquellos (enfermos y ancianos) que tienen menos recursos y/o mayor riesgo" (6), valor reflejado en el copago de las usuarias según su previsión.

Otro punto relevante de este estudio es la garantía de calidad, la cual no pudo ser evaluada debido a la no vigencia de dicha garantía en el momento de la realización del estudio y de la inexistencia de indicadores para su medición. La calidad es un aspecto fundamental planteado en la reforma en salud y como tal puede afectar todas las dimensiones contempladas en las GES, por lo cual se hace indispensable que las autoridades definan claramente que se entiende por estándares de calidad y cuales son los indicadores para su evaluación. Si bien se sabe que se está desarrollando el proceso de acreditación de los centros prestadores de salud que involucra el proceso de mejora continua de la calidad y de algunos procedimientos que forman parte de esta GES (13), este no es específico para evaluar la calidad de la atención en esta patología GES. Las sugerencias entregadas por los profesionales podrían ser un aporte para la medición de dicha garantía en este grupo de mujeres con esta patología en particular.

En cuanto al sistema de registros vigente, el alto desacuerdo refleja que este sistema no esta siendo una herramienta que cumple con las expectativas y necesidades de los profesionales, por lo cual es importante indagar en profundidad como podría facilitar el trabajo de los profesionales. El sistema de registros es fundamental para la navegación de las mujeres en el sistema y para el trabajo en red entre los diferentes niveles. Probablemente el desacuerdo se sustente en dificultades relacionadas con la alta demanda versus el tiempo requerido para completar los formularios requeridos (14), en un sistema definido por los mismos profesionales como arcaico. Existe evidencia de una mayor demanda (168\% superior a la estimada) entre los años 20052006 para la GES-CC; lo que derivó en una gran tensión en la red asistencial debido al tiempo requerido para el registro de estas atenciones (15). Además, a pesar de que este sistema dispone de un software computacional, no existe evidencia de 
su implementación en los tres niveles de atención $y$, antecedentes existentes en el MINSAL reconocen la necesidad de que probablemente deban ser realizarlos en forma manual por falta de recursos necesarios (14). Todas estas dificultades se vieron reflejadas en los aspectos negativos del sistema de registro que los profesionales describieron.

Actualmente no se cuenta con una evaluación global de la GES, lo que crea incertidumbre entre los profesionales, al momento de analizarla como una herramienta eficaz para el logro de los objetivos sanitarios planteados $(5,16)$. Es necesario por lo tanto conocer la efectividad de las prestaciones que el programa otorga para garantizar el cumplimiento de los objetivos sanitarios propuestos, disminuir las desigualdades en salud en el acceso, proveer servicios acordes a las expectativas de la población y reducir las tasas de mortalidad (5). Considerar la opinión de los profesionales involucrados directamente en la atención de las mujeres, es una vía válida para mejorar los estándares de calidad del programa.

\section{BIBLIOGRAFÍA}

1. Organización Panamericana de la Salud. Hoja informativa del programa mujer, salud y desarrollo, 2001. Hallado en: http://www.paho.org. Acceso: 5 de marzo de 2009.

2. Organización Panamericana de la Salud. Análisis de la situación del cáncer cervicouterino en América Latina y el Caribe, 2004. Hallado en: http://www.paho.org. Acceso: 5 de julio de 2007.

3. MINSAL. Mortalidad de los adultos por grupos de edad, 2003. Hallado en: http://deis.minsal.cl/deis/ev/ mortalidad_adulta/index.asp. Acceso: 10 de febrero de 2009.

4. Superintendencia de Salud. Qué es el Auge, 2009. Hallado en: http://www.supersalud.cl/568/propertyvalue-800.html. Acceso: 11 de marzo de 2009.

5. MINSAL. Objetivos sanitarios para la década 20002010, 2002. Hallado en: http://epi.minsal.cl/epi/html/ elvigia/vigia15.pdf \%20. Acceso: 20 de febrero de 2009.
6. MINSAL. Hacia un nuevo modelo de gestión en salud. Contenidos del proyecto de ley de autoridad sanitaria y gestión en salud y de medidas administrativas inmediatas, 2002. .Hallado en: http://www.ias.uchile. cl/files/biblioteca/gestion.pdf. Acceso: 10 de marzo de 2009.

7. MINSAL. Garantías Explicitas en Salud: Guía de Cáncer Cervicouterino, 2005. Hallado en: www.minsal.cl. Acceso: 25 de febrero de 2009.

8. Gonzalez F. Implementación del plan AUGE en pacientes con IRC. Rev Méd Chile 2003;131:545-51.

9. Ilabaca J. Redes en salud de la Reforma. 2007. Hallado en: http://www.nutricionistasdechile.cl/presentaciones/Redes_en_salud110507.pdf. Acceso: 9 de abril de 2009.

10. Bitrán R. Verificación del costo esperado por beneficiario del conjunto priorizado de problemas de salud con garantías explícitas 2005-2007. Resumen ejecutivo del informe final presentado al Ministerio de Salud de Chile. 2005. Hallado en: http://www.minsal.cl/ici/ destacados/estudio_verificacion/Resumen_Ejecutivo_GES_30_Noviembre_2005_Corregido.pdf. Acceso: 7 de abril de 2009.

11. Ley AUGE $N^{\circ} 19.966,2005$. MINSAL. Hallado en: http://www.supersalud.cl/normativa/571/articles-554_ recurso_1.pdf. Acceso: 5 de abril de 2009.

12. CEPAL. Protección social y sistemas de salud. La protección social: de cara al futuro, acceso, financiamiento y solidaridad, 2006. Hallado en: http://www. eclac.org/celade/noticias/paginas/5/27255/Cruces2. pdf. Acceso: 18 de julio de 2008.

13. Superintendencia de Salud. Estándares de acreditación 2007. Hallado en: http://www.supersalud.cl. Acceso: 15 de marzo de 2009.

14. MINSAL. Sistema Estadístico para el Sistema AUGE: Aplicación desde agosto de 2002. Hallado en: http:// deis.minsal.cl/deis/paso/manual\%20AUGE\%20piloto $\% 202002$.htm. Acceso: 18 julio de 2008.

15. Bastías G, Valdivia C. Reforma de Salud en Chile; el plan AUGE o Régimen de garantías explicitas en salud (GES). Su origen y evolución. 2007. Hallado en: http://escuela.med.puc.cl/publ/boletin/20072/ReformaSalud.pdf. Acceso: 9 de abril de 2009.

16. MINSAL. Proyecto de Ley sobre las Garantías en Salud. 2004. Hallado en: http://www.minsal.cl/ici/reforma_de_salud/AUGE\%20Resumen\%20Definitivo\% 2010\%20agosto\%202004.pdf. Acceso: 20 de Marzo de 2009. 